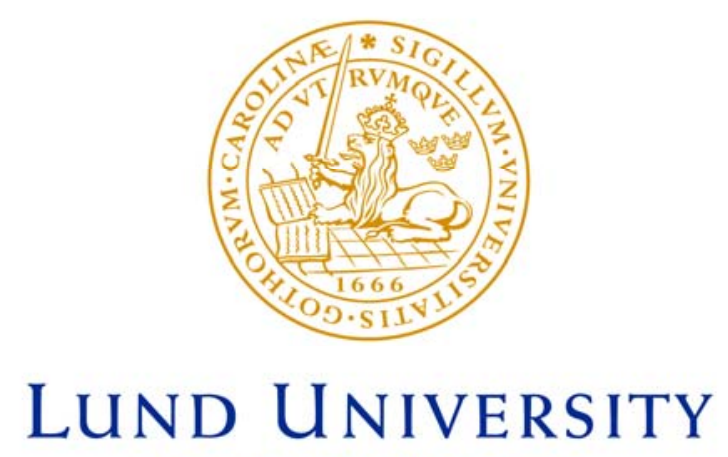

Faculty of Medicine

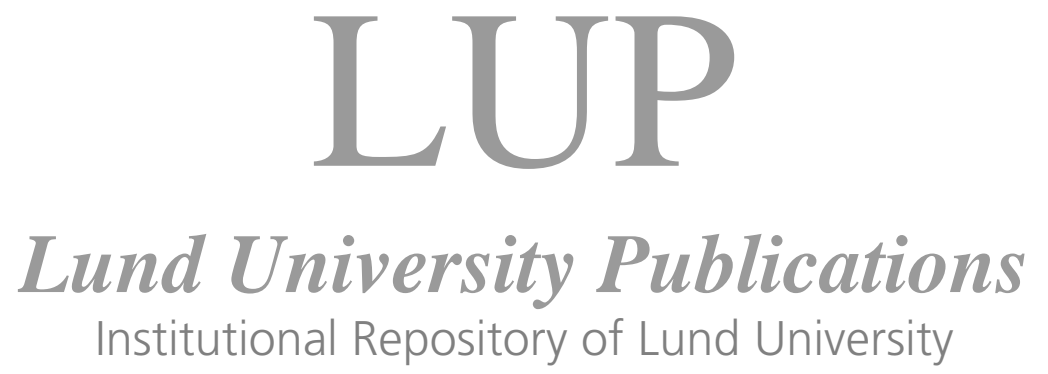

This is an author produced version of a paper published in Archives of gerontology and geriatrics. This paper has been peer-reviewed but does not include the final publisher proof-corrections or journal pagination.

Citation for the published paper:

Hans Brunnström, Lars Gustafson, Ulla Passant, Elisabet Englund

Prevalence of dementia subtypes: A 30-year retrospective survey of neuropathological reports

in Archives of gerontology and geriatrics, 2008, Issue: Aug 7

http://dx.doi.org/10.1016/j.archger.2008.06.005

Access to the published version may

require journal subscription.

Published with permission from:

Elsevier 


\title{
Prevalence of dementia subtypes: A 30-year retrospective survey of neuropathological reports
}

Hans Brunnström ${ }^{\mathrm{a}}$, Lars Gustafson ${ }^{\mathrm{b}}$, Ulla Passant ${ }^{\mathrm{b}}$, and Elisabet Englund ${ }^{\mathrm{a}, *}$

a Department of Pathology, University Hospital, S-221 85 Lund, Sweden
${ }^{\text {b }}$ Department of Psychogeriatrics, University Hospital, S-221 85 Lund, Sweden

* Corresponding author: Tel.: +46 46 173438; fax: +46 46143307.

E-mail address: elisabet.englund@skane.se (E. Englund)

\begin{abstract}
We investigated the distribution of neuropathologically defined dementia subtypes among individuals with dementia disorder. The neuropathological reports were studied on all patients ( $n=524$; 55.3\% females; median age 80, range 39-102 years) with clinically diagnosed dementia disorder who underwent complete autopsy including neuropathological examination within the Department of Pathology at the University Hospital in Lund, Sweden, during the years 1974-2004. The neuropathological diagnosis was Alzheimer's disease (AD) in $42.0 \%$ of the cases, vascular dementia $(\mathrm{VaD})$ in $23.7 \%$, dementia of combined Alzheimer and vascular pathology in 21.6\%, and frontotemporal dementia in $4.0 \%$ of the patients. The remaining $8.8 \%$ of the patients had other dementia disorders, including combinations other than combined Alzheimer and vascular pathology. The registered prevalence of dementia subtypes
\end{abstract}


depends on many variables, including referral habits, clinical and neuropathological judgments and diagnostic traditions, all of these variables potentially changing over time. This, however, does not seem to obscure the delineation of the major dementia subgroups. In this material of 30 years from Lund in the south of Sweden, AD by far dominated among dementia subtypes, while cerebrovascular pathology corresponded with the dementia disorder, either entirely or partly, in almost half of the demented patients.

Keywords: Alzheimer’s disease; Autopsy; Clinical diagnosis; Frontotemporal dementia; Vascular dementia 


\section{Introduction}

Dementia is a frequent disorder in elderly people, according to some of the more comprehensive epidemiological investigations affecting 6\% of the European (Lobo et al., 2000) and 7\% of the North American population (Krishnan et al., 2005) aged 65 years and older. In these large epidemiological studies, the major dementia subtypes were $\mathrm{AD}$ and $\mathrm{VaD}$. In the European study, $\mathrm{AD}$ represented $53.7 \%$ and $\mathrm{VaD} 15.8 \%$ of the patients, while the figures were $44.6 \%$ and $11.9 \%$, respectively in the American investigation. However, the dementia subtype diagnoses in the referred studies were based on clinical examination only, and not confirmed with postmortem neuropathology.

The accuracy of some clinical criteria for dementia subtypes have been evaluated in prospective clinico-pathological studies on populations of patients with various dementia subtypes (Jobst et al., 1998; Holmes et al., 1999; Lim et al., 1999). These studies, mainly focusing on $\mathrm{AD}$, showed that the clinical criteria for probable $\mathrm{AD}$ - as formulated by the NINCDS-ADRDA Work Group (McKhann et al., 1984), using the histopathological CERAD criteria (Mirra et al., 1991) as control - have a sensitivity in the range of $49-83 \%$ and a specificity spanning $55-100 \%$. One of the studies (Holmes et al., 1999) also presented corresponding analyses of $\mathrm{VaD}$ and dementia with Lewy bodies (DLB), indicating that, when correlating clinical diagnosis with subsequent neuropathologic analysis, the clinical criteria for these dementia subtypes have a lower sensitivity and a somewhat higher specificity.

These studies emphasize the importance of neuropathological examination for correct diagnosis of dementia subtype, as mismatching in clinical and histopathological 
phenotypes does occur. In other words, different histopathological features may cause a similar clinical condition in demented patients, while patients with different clinical conditions may have histopathological features of the same type. Therefore, it is important to distinguish between clinical and pathological principles for description of dementia, at least until etiology and pathogenesis is better understood, as has been suggested in reports from a long time-span (Roth, 1971; Gustafson, 1996; Mathuranath et al., 2000).

The aim of the present study was to investigate the prevalence of dementia subtypes, on the basis of neuropathological examination, from the reception area of the Lund University Hospital, Sweden.

\section{Material and methods}

\subsection{Study population}

In this retrospective study, all patients who died in the years 1974-2004 and fulfilled the following three criteria were included:

(1) A clinically diagnosed dementia disorder.

(2) A complete autopsy including neuropathological examination performed within the Department of Pathology at the University Hospital in Lund.

(3) A neuropathological condition in accordance with dementia disease.

In the very majority of the cases, the clinical diagnosis was transferred to the Department of Pathology via the autopsy referral - when missing (less than $2 \%$ of the patients) the medical records were consulted. Patients with clinical dementia where 
neuropathological examination did not reveal significant pathology (less than $1 \%$ of the cases during the studied years) were not included in the study.

\subsection{Data collection}

The neuropathological reports on all included patients $(n=524)$ were studied (i.e. the slides were not re-examined). Dementia subtype was recorded for each patient, the classification being in adherence with the Swedish consensus on dementia (Wallin et al., 1994). The subtypes were generally defined in accordance with criteria for AD, VaD and frontotemporal dementia (FTD) respectively (Braak and Braak, 1991; Mirra et al., 1991; Brun, 1994; The Lund and Manchester Groups, 1994), from the time they appeared.

\section{Results}

\subsection{Neuropathological diagnoses}

Of the 524 patients, the neuropathological diagnosis AD was found in 220 individuals, $\mathrm{VaD}$ in 124, dementia of combined Alzheimer and vascular pathology $(\mathrm{AD}+\mathrm{VaD})$ in 113 , and a diagnosis within the FTD group in 21 cases. The remaining 46 patients had other diagnoses, including combinations of dementia disorders other than combined Alzheimer and vascular pathology. Patient characteristics are presented in Table 1. 
The FTD group consisted of 14 patients with frontal lobe degeneration of nonAlzheimer type (FLD), 2 with motor neuron disease type of FTD, and 2 patients with Pick’s disease. Also, the group included 2 patients who exhibited the traits of corticobasal degeneration (CBD), and a single case that was classified as cortico-striatonigral degeneration.

The heterogeneous group 'other dementia disorders' included patients with dementia judged to be wholly or partially caused by trauma $\left(n=7^{1}\right)$, tumor $(n=5$, see footnote 1), Creutzfeldt-Jakob disease including Gerstmann-Sträussler-Scheinker syndrome $(n=4+1)$, hemodialysis $(n=4)$, alcohol abuse $(n=2)$, multiple system atrophy ( $n=2)$, post-surgical radiation $(n=2)$, herpes encephalitis $(n=1)$, Huntington's disease ( $n=1)$, multiple sclerosis $(n=1)$, neurosyphilis $(n=1)$, or vascular malformation $(n=1)$. Furthermore, there were three patients with dementia caused by a combination of Alzheimer pathology and frontal lobe degeneration, and one patient each with diffuse Lewy body disease without any other pathology, dementia caused by CBD combined with Alzheimer pathology, and dementia caused by progressive supranuclear palsy (PSP) combined with Alzheimer and vascular pathology. Also, there were nine patients with dementia of unresolved subtype.

${ }^{1}$ In one of the tumor patients, trauma also most likely contributed to the dementia disorder.

\subsection{Alzheimer and vascular pathology}


As stated above, 333 patients had either $\mathrm{AD}$ or $\mathrm{AD}+\mathrm{VaD}$, while 237 patients had $\mathrm{VaD}$ or $\mathrm{AD}+\mathrm{VaD}$. Among the 46 patients with 'other dementia disorders', Alzheimer and vascular pathology was considered to contribute to the dementia in 16 and 14 patients respectively. Thus, in total, 349 patients (66.6\% of all patients) had Alzheimer pathology of such degree that it was judged to contribute to the dementia disorder, while 251 (47.9\% of all patients) had vascular pathology of a similar, seemingly significant degree.

\subsection{Lewy body pathology}

Brainstem, subcortical and/or cortical Lewy bodies (LB) were reported in 37 of the patients with $\mathrm{AD}, 2$ of the patients with $\mathrm{VaD}$, and in 7 of the patients with $\mathrm{AD}+\mathrm{VaD}$. A presence of LB was not reported in any patient with FTD, while 3 patients with 'other dementia disorders' exhibited LB, including the one patient with widespread LB pathology as the sole finding (the only patient diagnosed as neuropathological DLB). Thus, 49 patients (9.4\% of all patients) had a presence of LB, with or without other Lewy related pathology such as Lewy neurites, and in the patients considered to have significant Alzheimer pathology, 12.9\% had concomitant LB.

\section{Discussion}

In this study we present the prevalence of different dementia subtypes based on the reports from neuropathological examination on a relatively large number of individuals with clinically diagnosed dementia. One of the criteria for inclusion in the study was 
complete autopsy performed at the Department of Pathology at the University Hospital in Lund. Deceased patients referred to the Department of Pathology from outside of the reception area for neuropathological examination (with autopsy at the local hospital) were thus excluded from this study. Consequently, the analysis may essentially represent the known population of demented patients, as defined within this region.

The study population of the present report partly overlaps that of an earlier longitudinal study from Lund, ongoing since 1968 (Brun and Gustafson, 1993). However, the populations differ through the strict adherence to autopsied cases in the present study, whereas diagnosed dementia cases from peripheral sites neuropathologically investigated in Lund - were included in the presentation from 1993. Furthermore, only early onset dementia cases were selected during the first phase of the previous Lund study.

In the present study, some potential sources of error can readily be identified:

(1) Selection bias: In Lund, patients with dementia have usually been diagnosed and followed up at primary care level or at the Department of Psychogeriatrics. Upon death, referral for autopsy has been particularly high from the latter, due to the mentioned Lund Longitudinal Dementia Study (Brun and Gustafson, 1993). In contrast, the autopsy rate in patients who have been followed up solely at a primary care level has been lower, and the selectivity, in this respect, may to some extent have influenced the proportional distribution of dementia subtypes at the Department of Pathology. Furthermore, patients with Parkinson's disease (PD) and other primary neurological/motor function diseases have usually been recognized and followed up at the Department of Neurology - dementia disorders are traditionally rarely handled within this specialty. From the former category, referral for autopsy upon death has 
been but exceptional. As the frequency of dementia is high in patients with PD (Cummings, 1988; Jellinger, 1997; Aarsland et al., 2003) and since this disease category by prevalence is not negligible, this referral bias is likely to have influenced the findings in the present study.

(2) Missed inclusion: In the present study the inclusion criteria were dementia disorder according to clinical records, as well as in accordance with neuropathological examination, and complete autopsy. It is unlikely that these criteria would erroneously have included any non-demented patients. However, there were nine patients with clinical records of dementia who underwent autopsy but not neuropathological examination during the investigated years, and also seven individuals that fulfilled all criteria, but in whom full pathological information was not retrievable. These cases were comparable to the whole group with regard to clinical diagnosis, age and sex, and their exclusion probably did not cause any significant imbalance of the study population.

(3) Neuropathological diagnostics: There are circumstances that may cause or contribute to a dementia syndrome but which normally are not revealed at pathological examination, such as alcohol abuse and vitamin B12 deficiency. Similarly, clinically detected normal pressure hydrocephalus typically manifests as central atrophy, for which many neuropathological diagnoses may apply. Furthermore, during the more than 30 years covered in the present study, the neuropathological procedures and classification of dementia disorders have changed, as in all major diagnostic centers world-wide. Here, for example, one patient was classified as exhibiting cortico-striatonigral degeneration, a diagnosis no longer used, while in 1999, the first case of CBD appeared, exhibiting the traits of a disease which to our understanding represents a 
similar, though not identical, disease. Also, there were nine patients with dementia of unresolved subtype, all autopsied in the 1970s and 1980s. Had these patients been examined today, more precise diagnoses most likely would have been reached, as staining and description of neuropathological findings have developed. We recognize the value of upgrading the entire study material to modern staining and classification criteria in the future.

The diagnosis $\mathrm{AD}+\mathrm{VaD}$ has, more importantly, remained constant over the years. Only patients with Alzheimer and vascular pathology of such degree that both were likely to have caused or contributed to the dementia were classified as $\mathrm{AD}+\mathrm{VaD}$, while those with significant Alzheimer pathology and a minor vascular component, such as a single minor infarction, were classified as AD.

From a neuropathological perspective, FTD denotes the diagnoses FLD, motor neuron disease type of FTD, and Pick’s disease (The Lund and Manchester Groups, 1994). Here, we also included CBD and PSP, considering the similarities of these two diseases to the FTD variants (Josephs et al., 2006), although no case of "pure” PSP was found in the study material. This is in accordance with the recently published consensus statement on frontotemporal lobar degeneration (FTLD) (Cairns et al., 2007), FTLD today being a commonly used umbrella term for the group.

On neuropathological grounds, the diagnosis DLB has repeatedly been considered but only rarely employed within our department. In order to examine the frequency of patients with Lewy related pathology as a potential contributing factor to dementia, the histopathological presence of LB, as recorded in the neuropathological reports, was noted in the present study. It is most likely that the presence of LB is underreported, as staining with antibodies against ubiquitin or $\alpha$-synuclein has not been routine during 
most years during the study period, and LB may be difficult to detect without either of these stains (Lennox et al., 1989; Spillantini et al., 1997), at least when the presence of LB is sparse.

In the present study, all but one of the patients identified with LB also had other significant dementia-related pathology, which is in accordance with a previous retrospective follow-up study on patients with clinical DLB (Londos et al., 1996). It is not known in how many of the patients the concomitant LB actually may have contributed to the dementia disorder. If regarding LB as suggested in the proposed DLB criteria (McKeith et al., 2005), 1.3\% of the cases in the present study material would be classified as neuropathological DLB, based on the reported pathological findings. However, the figure would be $7.2 \%$ if including only the patients examined 1998 or later (since the introduction of $\alpha$-synuclein staining at our department). Forthcoming studies will further address the issue of LB and synuclein pathology.

In the literature, there are several consecutive autopsy studies or investigations on comparable populations of demented patients neuropathologically examined upon death. However, only a few of these studies include a reasonably large number of patients and present detailed information on dementia subtypes. Table 2 demonstrates the distribution of demented patients among dementia subtypes in the 6 largest studies found (Galasko et al., 1994; Victoroff et al., 1995; Jellinger, 1996; Akatsu et al., 2002; Barker et al., 2002; Fu et al., 2004), together with the results of the present investigation. When needed, the study populations have been regrouped in order to facilitate comparison with the present study and with each other (with reservations due to sparse information concerning diagnostic considerations on some patient groups). However, in two of the studies (Galasko et al., 1994; Victoroff et al., 1995) it was not 
possible to separate patients with FTD from those with 'other dementia disorders'. The compared studies used different terminology for dementia associated with LB, such as dementia with Lewy bodies, diffuse Lewy body disease and Lewy body variant of Alzheimer's disease. In Table 2, the term dementia with Lewy bodies is used, representing patients with LB considered contributing to the dementia disorder, with or without concomitant Alzheimer pathology, excluding patients classified as PD with dementia or AD with PD. As previously discussed, the patients with LB pathology in our study have been classified depending on the major type of neuropathological findings, why these patients are found mainly in the groups $\mathrm{AD}$ and $\mathrm{AD}+\mathrm{VaD}$.

As evident from the listed dementia subtypes in all studies (Table 2), AD was the most common dementia subtype in all studies, followed by either DLB, VaD or AD + VaD. In our study VaD was the second most common diagnosis, closely followed by $\mathrm{AD}+\mathrm{VaD}$. The studies exhibit notable differences in frequency of DLB and VaD, the former representing $0.2-22.4 \%$ and the latter $2.4-23.7 \%$ of all cases. Although differences in study population, neuropathological examination, and subtype classification most likely have influenced these variations, it cannot be excluded that the differences also reflect a true ethnical and/or cultural diversity. However, we may conclude that the main dementia subtypes are largely comparable.

\section{Conflict of interest statement}

None. 


\section{Acknowledgements}

We wish to thank Annette Persson, Department of Pathology, and Helena

Andersson, Department of Psychogeriatrics, for valuable help, Prof. Em. Arne Brun,

Department of Pathology, for many years of solid work, and the Department of

Neurology for providing library facilities. The study was supported by the Swedish

Alzheimer foundation.

\section{References}

Aarsland, D., Andersen, K., Larsen, J.P., Lolk, A., Kragh-Sørensen, P., 2003. Prevalence and characteristics of dementia in Parkinson disease: an 8-year prospective study. Arch. Neurol. 60, 387-392.

Akatsu, H., Takahashi, M., Matsukawa, N., Ishikawa, Y., Kondo, N., Sato, T., Nakazawa, H., Yamada, T., Okada, H., Yamamoto, T., Kosaka, K., 2002. Subtype analysis of neuropathologically diagnosed patients in a Japanese geriatric hospital. J. Neurol. Sci. 196, 63-69.

Barker, W.W., Luis, C.A., Kashuba, A., Luis, M., Harwood, D.G., Loewenstein, D., Waters, C., Jimison, P., Shepherd, E., Sevush, S., Graff-Radford, N., Newland, D., Todd, M., Miller, B., Gold, M., Heilman, K., Doty, L., Goodman, I., Robinson, B., Pearl, G., Dickson, D., Duara, R., 2002. Relative frequencies of Alzheimer disease, Lewy body, vascular and frontotemporal dementia, and hippocampal sclerosis in the State of Florida Brain Bank. Alzheimer Dis. Assoc. Disord. 16, 203-212.

Braak, H., Braak, E., 1991. Neuropathological staging of Alzheimer-related changes. Acta Neuropathol. 82, 239-259.

Brun, A., 1994. Vascular dementia: pathological findings. In: Burns, A., Levy, R. (Eds.), Dementia. Chapman \& Hall, London, pp. 653-663.

Brun, A., Gustafson, L., 1993. The Lund longitudinal dementia study: a 25-year perspective on neuropathology, differential diagnosis and treatment. In: Corain, B., Iqbal, K., Nicolini, M., Winblad, B., Wisniewski, H., Zatta, P. (Eds.), Alzheimer's Disease: Advances in Clinical and Basic Research. John Wiley \& Sons Ltd., Chichester, pp. 3-18.

Cairns, N.J., Bigio, E.H., Mackenzie, I.R., Neumann, M., Lee, V.M., Hatanpaa, K.J., White, C.L., 3rd, Schneider, J.A., Grinberg, L.T., Halliday, G., Duyckaerts, C., 
Lowe, J.S., Holm, I.E., Tolnay, M., Okamoto, K., Yokoo, H., Murayama, S., Woulfe, J., Munoz, D.G., Dickson, D.W., Ince, P.G., Trojanowski, J.Q., Mann, D.M., Consortium for Frontotemporal Lobar Degeneration, 2007. Neuropathologic diagnostic and nosologic criteria for frontotemporal lobar degeneration: consensus of the Consortium for Frontotemporal Lobar Degeneration. Acta Neuropathol. 114, 5-22.

Cummings, J.L., 1988. Intellectual impairment in Parkinson's disease: clinical, pathologic, and biochemical correlates. J. Geriatr. Psychiatry Neurol. 1, 24-36.

Fu, C., Chute, D.J., Farag, E.S., Garakian, J., Cummings, J.L., Vinters, H.V., 2004. Comorbidity in dementia: an autopsy study. Arch. Pathol. Lab. Med. 128, 32-38.

Galasko, D., Hansen, L.A., Katzman, R., Wiederholt, W.,Masliah, E., Terry, R., Hill, L.R., Lessin, P., Thal, L.J., 1994. Clinical-neuropathological correlations in Alzheimer's disease and related dementias. Arch. Neurol. 51, 888-895.

Gustafson, L., 1996. What is dementia? Acta Neurol. Scand. 168, S22-S24.

Holmes, C., Cairns, N., Lantos, P., Mann, A., 1999. Validity of current clinical criteria for Alzheimer's disease, vascular dementia and dementia with Lewy bodies. Br. J. Psychiatry 174, 45-50.

Jellinger, K.A., 1996. Structural basis of dementia in neurodegenerative disorders. J. Neural. Transm. 47, S1-S29.

Jellinger, K.A., 1997. Morphological substrates of dementia in parkinsonism. A critical update. J. Neural. Transm. 51, S57-S82.

Jobst, K.A., Barnetson, L.P., Shepstone, B.J., 1998. Accurate prediction of histologically confirmed Alzheimer's disease and the differential diagnosis of dementia: the use of NINCDS-ADRDA and DSM-III-R criteria, SPECT, X-ray CT, and Apo E4 in medial temporal lobe dementias. Oxford Project to Investigate Memory and Aging. Int. Psychogeriatr. 10, 271-302.

Josephs, K.A., Petersen, R.C., Knopman, D.S., Boeve, B.F., Whitwell, J.L., Duffy, J.R., Parisi, J.E., Dickson, D.W., 2006. Clinicopathologic analysis of frontotemporal and corticobasal degenerations and PSP. Neurology 66, 41-48.

Krishnan, L.L., Petersen, N.J., Snow, A.L., Cully, J.A., Schulz, P.E., Graham, D.P., Morgan, R.O., Braun, U., Moffett, M.L., Yu, H.J., Kunik, M.E., 2005. Prevalence of dementia among Veterans Affairs medical care system users. Dement. Geriatr. Cogn. Disord. 20, 245-253.

Lennox, G., Lowe, J., Morrell, K., Landon, M., Mayer, R.J., 1989. Anti-ubiquitin immunocytochemistry is more sensitive than conventional techniques in the detection of diffuse Lewy body disease. J. Neurol. Neurosurg. Psychiatry 52, 67-71. 
Lim, A., Tsuang, D., Kukull, W., Nochlin, D., Leverenz, J., McCormick, W., Bowen, J., Teri, L., Thompson, J., Peskind, E.R., Raskind, M., Larson, E.B., 1999.

Cliniconeuropathological correlation of Alzheimer's disease in a community-based case series. J. Am. Geriatr. Soc. 47, 564-569.

Lobo, A., Launer, L.J., Fratiglioni, L., Andersen, K., Di Carlo, A., Breteler, M.M., Copeland, J.R., Dartigues, J.F., Jagger, C., Martinez-Lage, J., Soininen, H., Hofman, A., 2000. Prevalence of dementia and major subtypes in Europe: a collaborative study of population-based cohorts. Neurologic Diseases in the Elderly Research Group. Neurology 54, S4-S9.

Londos, E., Passant, U., Gustafson, L., Brun, A., 2001. Neuropathological correlates to clinically defined dementia with Lewy bodies. Int. J. Geriatr. Psychiatry 16, 667679.

Mathuranath, P.S., Xuereb, J.H., Bak, T., Hodges, J.R., 2000. Corticobasal ganglionic degeneration and/or frontotemporal dementia? A report of two overlap cases and review of literature. J. Neurol. Neurosurg. Psychiatry 68, 304-312.

McKeith, I.G., Dickson, D.W., Lowe, J., Emre, M., O’Brien, J.T., Feldman, H., Cummings, J., Duda, J.E., Lippa, C., Perry, E.K., Aarsland, D., Arai, H., Ballard, C.G., Boeve, B., Burn, D.J., Costa, D., Del Ser, T., Dubois, B., Galasko, D., Gauthier, S., Goetz, C.G., Gomez-Tortosa, E., Halliday, G., Hansen, L.A., Hardy, J., Iwatsubo, T., Kalaria, R.N., Kaufer, D., Kenny, R.A., Korczyn, A., Kosaka, K., Lee, V.M., Lees, A., Litvan, I., Londos, E., Lopez, O.L., Minoshima, S., Mizuno, Y., Molina, J.A., Mukaetova-Ladinska, E.B., Pasquier, F., Perry, R.H., Schulz, J.B., Trojanowski, J.Q., Yamada, M., Consortium on DLB, 2005. Diagnosis and management of dementia with Lewy bodies: third report of the DLB Consortium. Neurology 65, 1863-1872.

McKhann, G., Drachman, D., Folstein, M., Katzman, R., Price, D., Stadlan, E.M., 1984. Clinical diagnosis of Alzheimer's disease: report of the NINCDS-ADRDA Work Group under the auspices of Department of Health and Human Services Task Force on Alzheimer's Disease. Neurology 34, 939-944.

Mirra, S.S., Heyman, A., McKeel, D., Sumi, S.M., Crain, B.J., Brownlee, L.M., Vogel, F.S., Hughes, J.P., van Belle, G., Berg, L., 1991. The Consortium to Establish a Registry for Alzheimer's Disease (CERAD). Part II. Standardization of the neuropathologic assessment of Alzheimer’s disease. Neurology 41, 479-486.

Roth, M., 1971. Classification and aetiology in mental disorders of old age: some recent developments. In: Kay, D.W.K., Walk, A. (Eds.), Recent Developments in Psychogeriatrics: A Symposium. Headly Brothers, London, pp. 1-18.

Spillantini, M.G., Schmidt, M.L., Lee, V.M., Trojanowski, J.Q., Jakes, R., Goedert, M., 1997. a-Synuclein in Lewy bodies. Nature 388, 839-840. 
The Lund and Manchester Groups, Brun, A., Englund, E., Gustafson, L., Passant, U., Mann, D.M.A., Neary, D., Snowden, J.S., 1994. Clinical and neuropathological criteria for frontotemporal dementia. J. Neurol. Neurosurg. Psychiatry 57, 416-418.

Victoroff, J., Mack, W.J., Lyness, S.A., Chui, H.C., 1995. Multicenter clinicopathological correlation in dementia. Am. J. Psychiatry 152, 1476-1484.

Wallin, A., Brun, A., Gustafson, L., the Swedish Consensus Group, 1994. Swedish consensus on dementia diseases. Acta Neurol. Scand. 157, S1-31. 
Table 1

Characteristics for patients with different dementia subtypes and for all demented

\begin{tabular}{llll}
\hline Dementia subtype & $\begin{array}{l}\text { Patients } \\
{[\mathrm{n}(\%)]}\end{array}$ & $\begin{array}{l}\text { Sex } \\
\text { (\% female) }\end{array}$ & $\begin{array}{l}\text { Age } \\
\text { [median (range) }\end{array}$ \\
\hline $\mathrm{AD}$ & $220(42.0)$ & 59.5 & $79(55-96)$ \\
$\mathrm{VaD}$ & $124(23.7)$ & 43.5 & $81(52-102)$ \\
$\mathrm{AD}+\mathrm{VaD}$ & $113(21.6)$ & 61.1 & $83(67-97)$ \\
FTD & $21(4.0)$ & 71.4 & $73(52-86)$ \\
Other dementia & $46(8.8)$ & 45.7 & $74(39-87)$ \\
All demented & $524(100)$ & 55.3 & $80(39-102)$ \\
\hline
\end{tabular}




\section{Table 2}

Distribution of patients among pathologically defined dementia subtypes in some large studies (adjusted for facilitated comparison)

\begin{tabular}{lcclllll}
\hline Study, year & $\mathrm{n}$ & $\begin{array}{l}\mathrm{AD} \\
(\%)\end{array}$ & $\begin{array}{l}\mathrm{VaD} \\
(\%)\end{array}$ & $\begin{array}{l}\mathrm{AD}+\mathrm{VaD} \\
(\%)\end{array}$ & $\begin{array}{l}\text { DLB } \\
(\%)\end{array}$ & $\begin{array}{l}\text { FTD } \\
(\%)\end{array}$ & $\begin{array}{l}\text { Other } \\
(\%)\end{array}$ \\
\hline Galasko et al., 1994 & 170 & 56.5 & 2.4 & 7.1 & 22.4 & $\mathrm{a}$ & 11.8 \\
Victoroff et al., 1995 & 196 & 44.9 & 4.6 & 12.8 & 6.6 & $\mathrm{a}$ & 31.1 \\
Jellinger, 1996 & 540 & 65.0 & 8.5 & 4.1 & 6.1 & 2.8 & 13.5 \\
Akatsu et al., 2002 & 158 & 46.2 & 21.5 & 5.7 & 17.7 & 3.2 & 5.7 \\
Barker et al., 2002 & 382 & 41.6 & 3.1 & 11.3 & 22.0 & 4.7 & 17.3 \\
Fu et al., 2004 & 202 & 63.9 & 5.9 & 2.5 & 11.9 & 4.0 & 11.9 \\
Present study & 524 & 42.0 & 23.7 & 21.6 & $0.2^{\mathrm{b}}$ & 4.0 & 8.6 \\
& & & & & & &
\end{tabular}

Notes: ${ }^{\mathrm{a}}$ Included in Other dementia disorders, ${ }^{\mathrm{b}}$ Mainly included in AD and AD + VaD (see text for details). 